\title{
Development of silicate hollow core photonic crystal fiber
}

\author{
Dariusz Pysz, ${ }^{* 1}$ Ryszard Stępień, ${ }^{1}$ Ireneusz Kujawa ${ }^{1}$, Rafał Kasztelanic, ${ }^{2}$ Tadeusz Martynkien, ${ }^{3}$ \\ Francis Berghmans, ${ }^{4}$ Hugo Thienpont ${ }^{4}$ and Ryszard Buczyński ${ }^{1,2}$ \\ ${ }^{1}$ Glass Laboratory, Institute of Electronic Materials Technology, 01-919 Warsaw, Poland, \\ ${ }^{2}$ Faculty of Physics, University of Warsaw, 02-093 Warsaw, Poland \\ ${ }^{3}$ Institute of Physics, Wroclaw University of Technology, 50 - 370 Wroclaw, Poland \\ ${ }^{4}$ Dep. of Appl. Physics and Photonics, Vrije Universiteit Brussel, B-1050, Brussels, Belgium
}

Received March 20, 2010; accepted March 29, 2010; published March 31, 2010

\begin{abstract}
In this paper we report on fabrication and characterization of a large core hollow core photonic crystal fiber. The fiber is designed and modeled using a finite element method taking into account material dispersion of the glass. A sodium-calcium silicate glass SK222 is used for photonic crystal fiber development. The hollow core is made by omitting 37 central capillaries and it has the diameter of $26 \mathrm{~mm}$. The hexagonal photonic cladding is composed of 12 rings with the lattice pitch of $2.8 \mu \mathrm{m}$ with the filling factor of 0.86 . We have measured a large band gap in the range $560 \div 670 \mathrm{~nm}$ with a center wavelength at $630 \mathrm{~nm}$ for the measured fiber.
\end{abstract}

Hollow core photonic crystal fibers (PCF) are one of the most interesting types of fibers with unique properties of light guidance in the air. A photonic band gap effect in the periodic structure of the photonic cladding allows light guidance in the low index core $[1,2]$.

Hollow core PCFs allow a reduction of all phenomena related to the interaction between glass and light, since most of energy propagates in the air. Therefore dispersion, multiphoton absorption, Raman scattering and other nonlinear effects are dramatically reduced. A silica based hollow core PCF achieves a very low attenuation of $1.2 \mathrm{~dB} / \mathrm{km}$ for $1.62 \mu \mathrm{m}$ [3]. In this case $99,5 \%$ of energy in the fundamental mode propagates in the air. Further reduction of the attenuation to gain the attenuation level of standard telecommunication optical fibers at $0.1 \mathrm{~dB} / \mathrm{m}$ is uncertain because surface modes are created at the glass-air border near the core. Since their propagation constants can be very similar to the fundamental guiding mode, the modes can be coupled and energy is transferred from the fundamental to the surface modes [4].

Hollow core PCFs made of soft glasses witness higher attenuation since the attenuation of glass itself is much higher. However, due to their higher refractive index they are attractive components from which to build a new type of devices infiltrated with polymer or liquids e.g. liquid crystals [5]. In this paper we present the development and characterisation of a hollow core PCF with a large hollow core based on silicate glass.

\footnotetext{
*E-mail: dariusz.pysz@iitme.edu.pl
}

For the PCF fabrication a sodium-calcium silicate glass SK222 (68.4\% SiO2, 2.4\%A12O3, 2\% B2O3, 12.3\% $\mathrm{Na} 2 \mathrm{O}, 0.7 \% \mathrm{~K} 2 \mathrm{O}$ ) is used. The SK222 glass is an ideal test bed material for the development of fabrication technology of soft glass based PCFs due to its low costs and very good rheological properties. On other hand, the attenuation in the glass as high as $15 \mathrm{~dB} / \mathrm{m}$ at $\lambda=1,55 \mu \mathrm{m}$. The properties of SK222 glass investigated by the differential thermal analysis are shown in Table 1.

\begin{tabular}{|l|r|}
\hline \multicolumn{1}{|c|}{ Glass type } & SK222 \\
\hline Refractive index $\mathrm{n}_{\mathrm{D}}$ & 1.520 \\
\hline $\begin{array}{l}\text { Linear expansion coefficient for } \\
\text { temperature range: } 20 \div 450^{\circ} \mathrm{C}\left[10^{-7} \mathrm{~K}^{-1}\right]\end{array}$ & 89.0 \\
\hline Transition ttemperature $\mathrm{Tg}\left[{ }^{\circ} \mathrm{C}\right]$ & 542 \\
\hline $\begin{array}{l}\text { Dylatometric softening temperature } \\
\text { DST }\left[{ }^{\circ} \mathrm{C}\right]\end{array}$ & 610 \\
\hline
\end{tabular}

Tab.1. Thermal properties of SK222 glass

A hollow core PCF labeled PBF3 is successfully fabricated using a stack-and-draw technique.

The structure of the fiber is designed as a hexagonal lattice with 31 capillaries on the diagonal. The hollow core is created by omitting 37 central capillaries. Therefore the photonic cladding is built by 12 rings of holes. The thickness of a glass layer surrounding the hollow core is decreased to a level of $(\Lambda-d) / 2$ to reduce losses related to surface modes. The design of the fiber structure and cross section of a developed subpreform is presented in Fig. 1. 


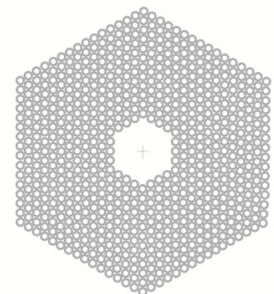

a)

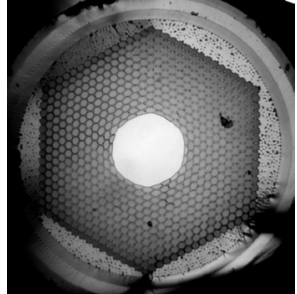

b)
Fig.1. The hollow core fiber labeled PBF3. a) a design of the fiber structure, $b$ ) the cross section of the subpreform

A correct structure without deformations and brakes is achieved in the subpreform. Using this subpreform a few series of hollow core PCFs are drawn with a diameter in the range $120 \div 200 \mu \mathrm{m}$ (Fig.2 and Fig. 3). The technological challenge of this fabrication is related to influence control of drawing process parameters on a structure filling factor, holes and core sizes and regularity of the crystal lattice. The final PCFs have a regular structure and the filling factor in the range 0.84-0.93. The thickness of glass walls surrounding the hollow core is lower than that in the photonic cladding (Fig. 2c, 3c). The hexagonal and kagome lattices obtained are based on the same preform with various drawing conditions (Fig.2 and Fig. 3). The hollow core diameter is at the $30 \mu \mathrm{m}$ level for both types of lattices. The structure parameters of selected PCFs are presented in Table 2.

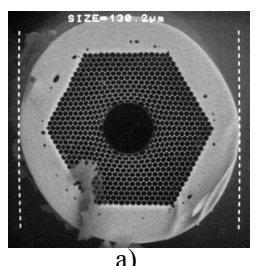

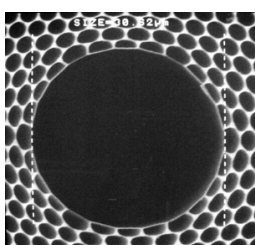

b)

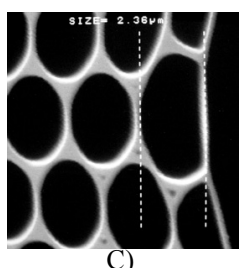

C)
Fig.2. Hollow core PCF with hexagonal lattice labeled PBF3A2: a) the cross-section of the fiber, b) the hollow core, c) the photonic cladding

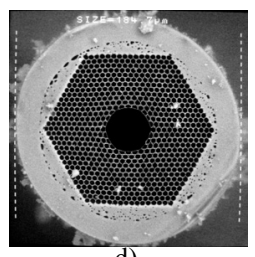

d)

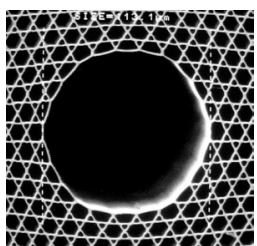

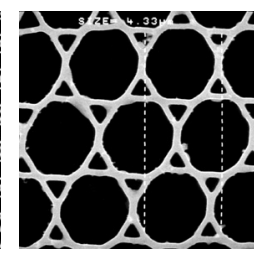

f)
Fig.3. Hollow core PCF with kagome lattice labeled PBF3B1: a) the cross-section of the fiber, b) the hollow core, c) thephotonic cladding

The hollow core PCF PBF3A2 is further selected for optical characterization. A broadband supercontinuum is used as light source in the measurement setup. It covers range from 500 to $1100 \mathrm{~nm}$. A spectrum of this supercontinuum source is presented in Fig.4. The collimated supercontinuum beam is coupled into the hollow core PCF with a 40x microscope objective. The output signal is delivered to spectrometers (VIS and NIR range: $400-1100 \mathrm{~nm}$ ) by a multimode optical fiber. The measurement setup is presented in Fig.5.

\begin{tabular}{|l|c|c|}
\hline & PBF3A2 & PBF3B1 \\
\hline Fiber diameter $[\mu \mathrm{m}]$ & 130 & 185 \\
\hline Diameter of photonic cladding $[\mu \mathrm{m}]$ & $98-103$ & 139 \\
\hline Core diameter $[\mu \mathrm{m}]$ & $30-31$ & 36.5 \\
\hline Wall thickness round the core $[\mu \mathrm{m}]$ & $0,15-0,2$ & $0,16-0,2$ \\
\hline $\begin{array}{l}\text { Walls thickness within photonic } \\
\text { cadding }(\Lambda \text {-d) }[\mu \mathrm{m}]\end{array}$ & 0,22 & 0,4 \\
\hline The diameter of the holes $(\mathrm{d})[\mu \mathrm{m}]$ & 2,75 & 3.93 \\
\hline Lattice constant $(\Lambda)[\mu \mathrm{m}]$ & 2,97 & 4.33 \\
\hline Filling factor $(\mathrm{d} / \Lambda) \square$ & 0,93 & 0,91 \\
\hline
\end{tabular}

Tab.2. Geometrical parameters of PBF3 hollow core PCFs.

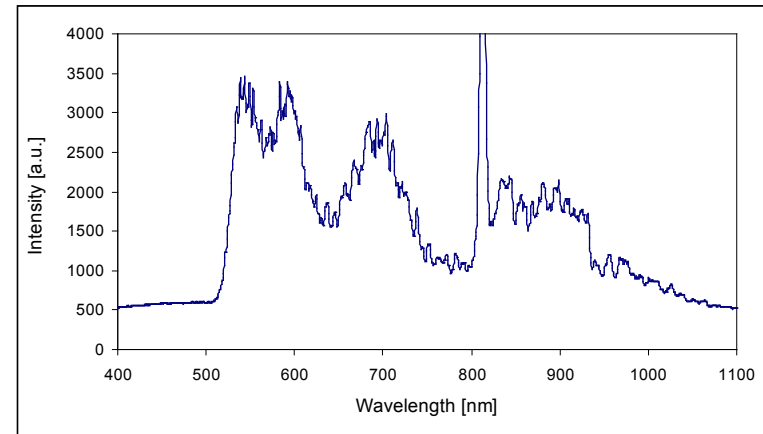

Fig.4. A spectrum of the supercontinuum source used in the experiment setup. A high pick for $\lambda=805 \mathrm{~nm}$ denotes a pump peak.

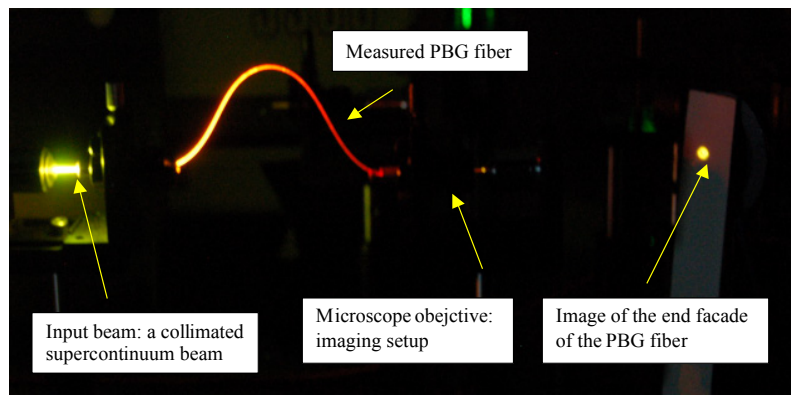

Fig.5. The setup for transmission and attenuation measurements in the hollow core PCFs.

An imaging system composed of a microscope objective and magnifying lens is built behind the hollow core fiber. The system allows observing a near-field distribution at the end facet of the fiber and is used for the optimisation of fiber coupling. With the imaging system we can actively align the fiber with respect to the laser 
beam to selectively couple light into the core without excitation of the fiber cladding (Fig. 6).

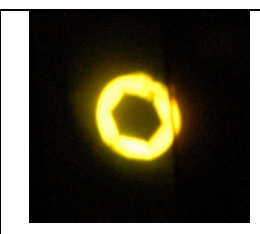

a)

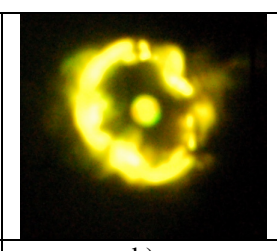

b)

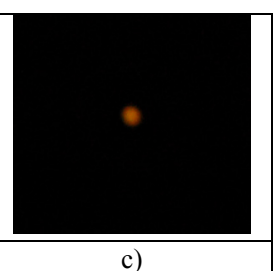

c)
Fig.6. Alignment procedure for transmission measurement in the hollow core PCFs. The near field distribution and the end facet of the hollow core fiber PBF3A2.: a): step1: light is coupled into the fiber cladding, b) step2: light is coupled into the fiber cladding and the hollow core, c) step 3 : light is coupled into the hollow core.

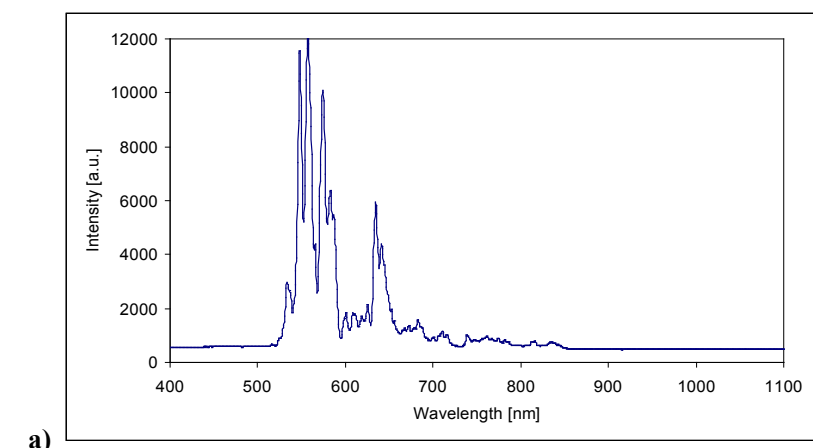

a)

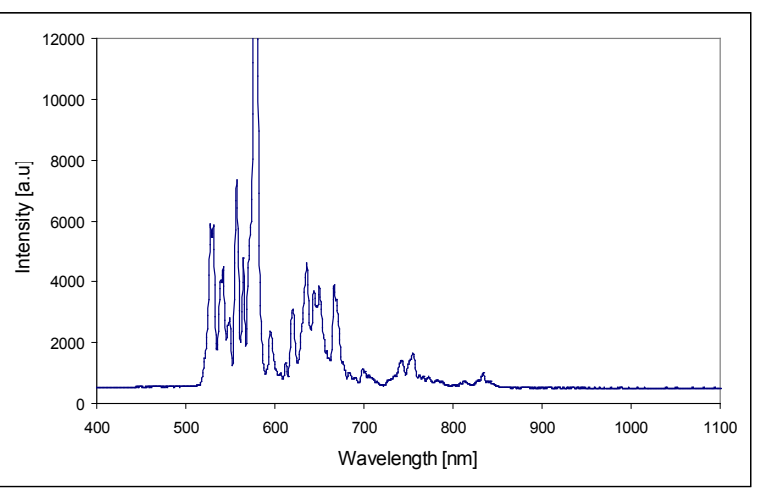

b)

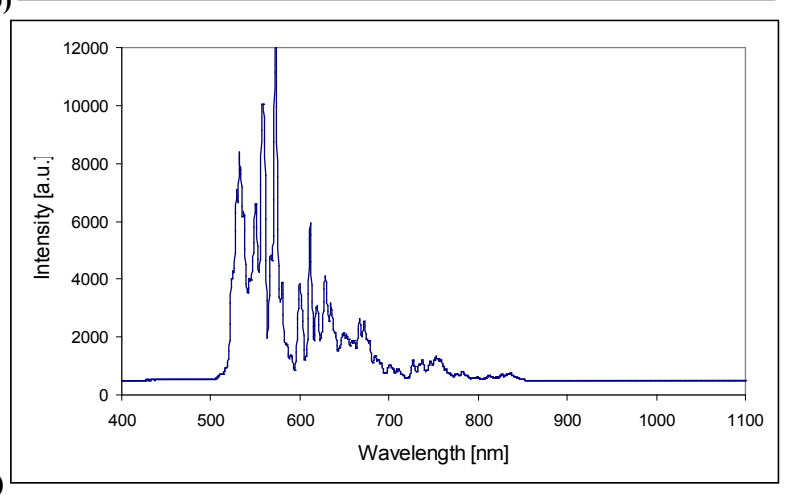

Fig.7. Measurements of transmission spectra in a hollow core of the PBF3A2 fiber. For different lengths: a) $103 \mathrm{~cm}$, b) $38 \mathrm{~cm}$, c) $32 \mathrm{~cm}$.
Measurement results of transmission of the broadband spectrum in PBF3A2 fiber are shown in Fig.7. The guidance of light in the range $560-670 \mathrm{~nm}$ is observed in a fiber sample $103 \mathrm{~cm}$ long. Similar spectra ranges are also registered for shorter samples -38 and $32 \mathrm{~cm}$. The constant range of guided spectra demonstrates the position of a photonic band gap and good homogeneity of the fabricated structure. The experimental results are in good agreement with the modeling of the considered fiber performed with the finite element method (Fig. 8).

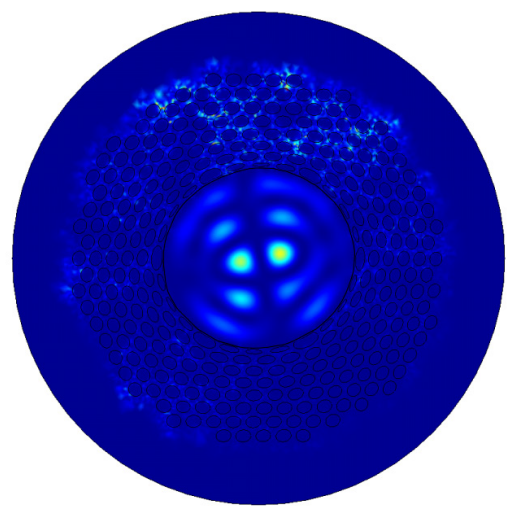

Fig.8. Calculated intensity distribution in the hollow core PCF PBF3A2 at $\lambda=635 \mathrm{~nm}$.

The transmitted spectra are measured for a different length of the fiber sample with the same coupling conditions. Using cut-back technique attenuation of $2.1 \mathrm{~dB} / \mathrm{m}$ is defined. This value is very high with respect to reported pure silica hollow core PCFs [3]. However, it is drastically smaller than the attenuation of bulk SK222 glass used for the PCF fabrication and proves good light confinement in the air core and week coupling to the surface modes..

This work was supported by the Polish Ministry of Science and Higher Education grant 3T11B 07230.

\section{References}

[1] R. F. Cregan, B. J. Mangan, J. C. Knight, T. A. Birks, P. S. Russell, P. J. Roberts, D. C. Allan: Single-mode photonic band gap guidance of light in air, Science 285, 1537-1539 (1999).

[2] A. Bjarklev, J. Broeng, A. S. Bjarklev, Photonic Crystal Fibres, Springer, |Berlin, 2003.

[3] P. J. Roberts at al., "Ultimate low loss of hollow-core photonic crystal fibres," Opt. Express 13, 236-244 (2005).

[4] K. Saitoh, N. A. Mortensen, and M. Koshiba, "Air-core photonic band-gap fibers: the impact of surface modes," Opt. Express 12, 394-400 (2004).

[5] S. Ertman, T. R. Wolinski, D. Pysz, R. Buczynski, E. NowinowskiKruszelnicki, and R. Dabrowski, "Low-loss propagation and continuously tunable birefringence in high-index photonic crystal fibers filled with nematic liquid crystals," Opt. Express 17, 1929819310 (2009). 